\title{
USING A HUMANOID ROBOT AS THE PROMOTER OF THE INTERACTION WITH CHILDREN IN THE CONTEXT OF EDUCATIONAL GAMES
}

\author{
${ }^{1}$ Helder Freitas, ${ }^{2}$ Pedro Costa, ${ }^{3}$ Vinícius Silva, \\ ${ }^{4}$ Ana Paula Pereira, ${ }^{5}$ Filomena Soares, 6 João Sena Esteves \\ 1,2 Department of Industrial Electronics, University of Minho, Guimarães, Portugal \\ 3,5,6R\&D Centre Algoritmi, University of Minho, Guimarães, Portugal \\ ${ }^{4}$ Education Research Centre, Institute of Education, University of Minho, Braga, Portugal \\ a68580@alunos.uminho.pt,a68535@alunos.uminho.pt \\ a65312@alunos.uminho.pt, appereira@ie.uminho.pt \\ fsoares@dei.uminho.pt,sena@dei.uminho.pt
}

\begin{abstract}
Society should care about those with special needs. Part of a proper care involves the development of new technologies and devices aiming at improving their quality of life. Research conducted at universities on this subject should be followed by the industrial development of commercial products and governmental institutions may play an important role by establishing conditions ensuring that the results are made available to those who need them. This paper presents the details of a system, still at the early stages of research level, aimed at helping children with Autism Spectrum Disorder (ASD). It uses ZECA, a humanoid robot Zeno R-50, acting as the promoter of the interaction with children, by teaching colours and geometric figures in the context of two educational game scenarios: identification of five geometric figures and identification of five colours. So far, the system was tested in a school environment with typically developing children, in order to validate the experimental setup and the game design. The results obtained in these tests allowed optimizing the system before starting the work in elementary schools with children with ASD, which is the next step in the research.
\end{abstract}

Keywords: Human-Robot Interaction, Educational Games, Autism Spectrum Disorder, ZECA robot, Geometric Figures, Colours.

\section{Introduction}

Human-Robot Interaction (HRI) is an important topic in the scientific community. HRI tries to build a bridge between the machines and humans, where "intelligent" robots are used to improve the quality of life of a human being. The general goal is to promote collaboration between humans and robots that can autonomously perform tasks and effectively communicate their "intentions". Recently, HRI has been a present topic in Autism Spectrum Disorder (ASD) research, where social robots are being used to help individuals with special needs in their daily activities [1]. Besides having difficulties in social communication [2], individuals with ASD are characterized by displaying repetitive patterns of behaviour, for having restricted activities or interests, and impairments in social communication.

Moreover, they have difficulties in putting themselves into someone else's perspective and being aware of how to react to another's feelings, in real time, i.e., they have difficulty to show empathy
[3]. In general, these difficulties in interpreting social situations, causes children with ASD to lose or miss information on what is happening or happened during the social interaction [4].

There is a strong contrast in the development of children with ASD when compared with typically developing children, and therefore they need timely and adequate intervention that addresses to all of their impaired areas of development. Thus, different approaches are used in intervention processes with children with ASD, by tackling individually each impairment that these children present. More recently, the use of social robots has been explored in order to facilitate intervention process of children with ASD.

The robot physical appearance plays an important role in the interaction process with a person. There is a range of different designs of robots that are used in intervention with children with ASD, varying from simple designs, e.g. fourwheeled mobile robots, to many levels of anthropomorphic forms, including humanoid [5], animal-like [6], and machine-like systems [7]. 
Therefore, it is important to discuss the appropriate features a robot must have, in order to be used in intervention process with children with ASD. A humanoid robot promises a great potential for generalization [5], being a useful tool to develop social-emotional skills in the intervention of children with ASD. For example, imitation and emotion recognition activities can be harder if the robot does not present a human form [8].

KASPAR, a minimally expressive child-sized robot [9] was used as a social mediator between the child and the experimenter as well as a teaching tool in a study to verify if the robot could help children with ASD to learn appropriate physical social engagement. Besides promoting social engagement, an additional goal of this study was to understand if the robot could facilitate the ability to acquire knowledge about human body parts. KASPAR is equipped with tactile sensors which allow the automatic response to gentle or harsh touches from the child. The results showed that children who initially were not able to identify some of the body parts, showed an improvement of their knowledge. Additionally, the robot was a useful tool to promote appropriate tactile interaction since gentle touches on the robot were always lower than harsh touches along the sessions.

Another approach of using social robots with children with ASD consisted in using NAO [10] in intervention sessions. The accomplishment of this study helped to prove how valuable this robot can be in helping children with ASD to develop their social skills [11]. Children who had difficulties in interacting with their peers, liked NAO and considered it as a friend. One of the activities that children enjoyed doing was playing the game of animals. In this game, NAO sits in front of the child and asks for a card of a given animal, then the child chose a card and places it in front of NAO. If the child has selected correctly, NAO reinforces the child positively. If the answer is incorrect, the body language is subdued and it gives the child a consoling but encouraging message.

FACE, a work developed by Mazzei et al. [12], consisted in a first stage in developing a humanoid robot to allow children with ASD to deal with expressive and emotional information. This robot, FACE, has 32 servo motors that allow it to move its artificial skin, enabling human facial expressions to be re-created. The system was tested with five children with ASD and fifteen typically developing children. The activities that were conducted with the children consisted in labelling the six basic facial expressions [13] (happiness, sadness, anger, fear, surprise, and disgust). The results showed that happiness, sadness, and anger were correctly labelled with high accuracy for both children with ASD and typically developing children. Conversely, the facial expressions of fear, disgust, and surprise were not correctly labelled, particularly by participants with ASD.

Sandra Costa [5] presented a child-robot interaction study, where children had to recognize and label emotions displayed by ZECA robot in two different game scenarios, involving imitation and storytelling activities. The goal of these scenarios was to help a child with ASD to acquire knowledge about different emotions and to improve their recognition skills. The Imitate Me game scenario consisted in ZECA displaying a facial expression, and then asking the child to show the same facial expression. The experimenter used a wireless keypad to classify the answer as either successful or unsuccessful. In the Storytelling game scenario, social stories were told by ZECA, and the child had to identify the emotion felt by the character, in this case ZECA, in each story. In general, the results show that the children accomplished a good performance. In addition, and as expected, the storytelling game scenario provided more difficulties to the children as they have difficulties in recognizing emotional states in the others.

There are various implementations of HRI systems in the literature. However, few of these systems targets individuals with ASD, especially children. Therefore, few projects world-wide pursue to include robots as part of the intervention program for individuals with ASD [14]. These studies have demonstrated that robots can promote a high degree of motivation and engagement in subjects, including subjects who are improbable or reluctant to interact socially with human professionals [15]. However, most of them focus on promoting social skills of recognizing and labelling emotions as well as the recognition of body parts, with fewer focusing on transmitting academic skills, for example, the recognition of geometric shapes, numbers, colours, among others.

Following this trend, the present work concerns the development of an interactive environment with a humanoid robot Zeno $R-50$ [16]. This environment contains activities that promote the development of skills, in this particular case, the recognition and labelling of geometric figures and colours. The paper is organized as follows: Section II presents the overall system and the methodology followed. The results and their discussion are addressed in Section III. Section IV finalizes the article, with the conclusions and future work.

\section{Methodology}

This section introduces the project and work in progress goals as well as experimental setup. There is also an explanation of the designed activities for the interaction child-robot. 
The developed interface that controls the environment and stores the information acquired from the activities is then presented. In the end, the characterization of the experimental sessions with the children are detailed.

\section{- Project and Work in progress goals}

The main goal of the research project where the work presented in this paper is included, is to develop an interactive environment capable of promoting academic and social skills, aimed at helping children with ASD. The promoter of the interaction with children is ZECA, a humanoid robot Zeno $R-50$ which establishes a dialogue with the child, congratulating the child and/or transmitting positive reinforcement. The system evaluates the child's behaviour during a session, in order to find out if the interaction promoted by the robot somehow affects positively the child's competence.

Specifically, in the work presented in this paper the goal was to design and develop two educational game scenarios for teaching colours and geometric figures: recognition of five geometric shapes (square, triangle, rectangle, circle, and pentagon) and recognition of five colours (red, blue, green, black, and yellow). These activities should promote the development of social and academic skills in typically developing children attending the kindergarten.

\section{- Environment}

The environment setup to interact with the child is presented in Figure 1. It is composed of the ZECA robot, a PlayStation Eye Camera, and a computer.

Each of these components has a fundamental role: the PlayStation Eye Camera that is connected to the computer, is responsible for capturing the frames of the game board; the computer analyses and processes them through image processing algorithms to recognize the geometric figures and colours; and ZECA robot promotes the interaction with the child.

ZECA, as the promoter of the interaction with the child, has a friendly dialogue with him/her. It starts by greeting the child and by explaining the activity's instructions. During the activity, the robot keeps on giving feedback about each move. It congratulates the child when he/she responds correctly; otherwise it transmits a positive reinforcement. This feedback corresponds to commands sent by the computer, which are interpreted by the robot and transmitted through voice messages, facial expressions and/or movements.

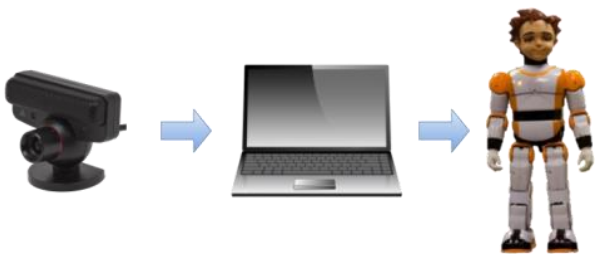

Figure 1: Proposed environment: PlayStation Eye Camera, a computer and the ZECA robot.

\section{- Activities}

Two activities were developed, namely: the identification activity of geometric figures and the identification activity of colours. Both activities have two levels of difficulty.

The geometric figures activity consists on the identification of geometric figures being: the square, the rectangle, the triangle, the circle, and the pentagon. The first level consists on completing the game board, Figure 2, where the drawing and the name of each geometric figure is presented. These geometric figures are positioned randomly on the board. So, there are other boards with the same geometric figures placed in other positions. There is more than one board so that children do not memorize the position where they should put the game pieces.

The second level has an empty board, without the drawings or names of the geometric figures. ZECA robot asks for a geometric figure and the player must place the corresponding piece on the board.

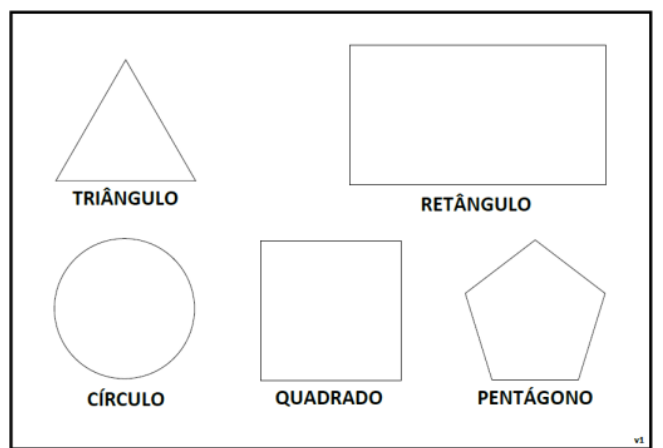

Figure 2: Board for the Geometric Figures Activity (names written in Portuguese).

At the end of each move, ZECA robot gives a feedback to the player. In the first level, the piece must be identified and its position compared with the position of the same geometric figure in the board. For positive feedback, the two positions should be, approximately, in the same position on the board. Otherwise, the robot encourages the child to try again.

In the second level, the piece played is identified and it is verified if it corresponds to the geometric figure requested by ZECA. 
When the geometric figure placed by the child matches the one asked by the robot, there is a positive feedback, and the robot congratulates the child. Otherwise, the feedback is a positive reinforcement.

The colours activity consists on the identification of colours blue, red, green, yellow, and black. The first level of the colours activity is similar to the first level of the geometric figures. It is also a matching activity. The board, Figure 3 , has three of the five game colours and their respective name. To play with all game colours it is necessary to at least play with two different colour boards, because each one has only three colours.

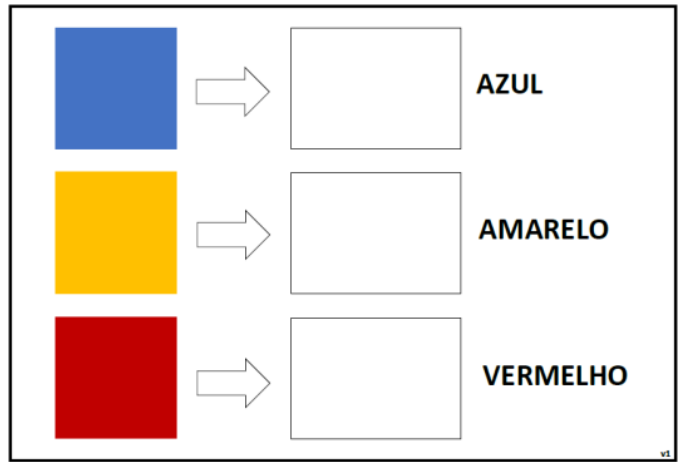

Figure 3: Board for the Colour Activity.

The second level of the colours activity similar to the second level of the geometric figures activity. In this case, the colour is requested by ZECA and the player must place the corresponding colour piece on the board.

To differentiate the colour in the board with the game pieces in the first level of colour activity, it was defined that the board colours would be squares and the game pieces would be rectangles.

At the end of each move, ZECA gives a feedback to the player. In the first level, the colour of the piece must be identified and compared to the position of the same colour in the board. In order to obtain a positive feedback, the player must place the piece in the rectangle in front of the board colour. If the piece is placed outside of the correct rectangle or another colour is placed in that rectangle a negative feedback is obtained.

In the second level, the colour of the piece played is identified and it is verified if it corresponds to the colour requested by the robot. The positive feedback is achieved when the both correspond to the same colour. Otherwise it is achieved a reinforcement feedback.

\section{- Interface}

The interface developed is called LCFZ (Learn Colours and Figures with ZECA) and it was created using the development tool Qt Creator.
The main goal of the LCFZ is for the user to have control of all the environment setup at any time. It allows calibrating the system vision, registering the participant, selecting the activity, and obtaining a record with all the information gathered during the activity, along with the answers given by the participants, as well as obtaining a graphic record of the performance in each activity.

The LCFZ is divided in two windows, the "Main" and the "Information" window. The "Main" window, Figure 4, is responsible for: controlling the vision system; establishing a communication with the robot ZECA; and to choose the activity and then to start or stop it. The "Information" window is connected to the participants, where they can be registered, selected, and see their performance.

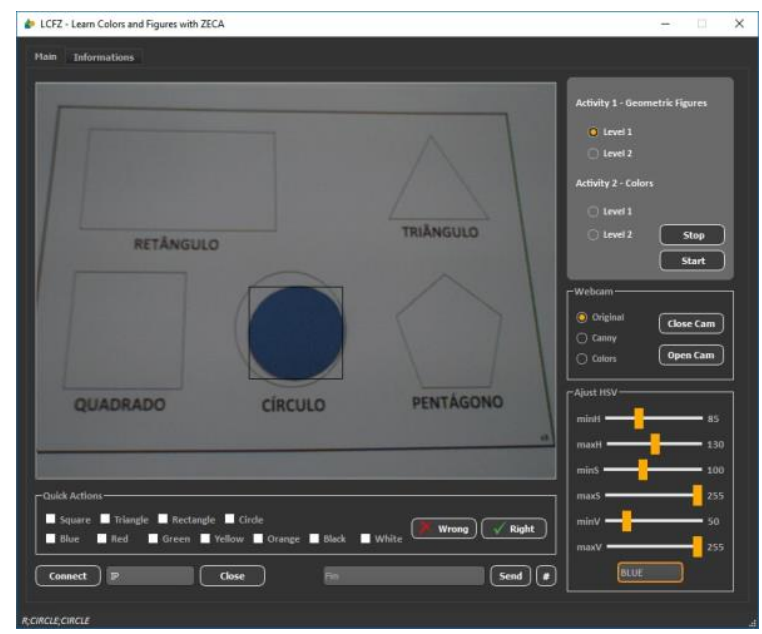

Figure 4: Main window of LCFZ.

The calibration of the vision system is performed through the "Main" window. The detection of each colour can be improved by the adjustment their Hue-Saturation-Value parameters (HSV). So, when it is necessary, the user, before starting an activity, selects the colour from the combo box present in the block "Adjust HSV" and then adjusts the slide bars for the colour. To improve this adjustment, in the "Webcam" block the user can select the option "Colours" where the displayed image is changed for a binary one, only white and black, where the white section is the colour that it is being filtered and calibrated.

A connection must be established between the LCFZ and the robot. For this purpose, there is a section that contains two buttons, the "Connect" button to establish the communication and the "Close" button to close the previously established connection. It also has a text box where the IP address can be changed.

After selecting a child, and only after that, the activity can be chosen in the upper right block, and the user can start or stop the activity.

The other window is called "Information", and it is where the information of each participant is 
presented and managed. Each section of "Information" window will be shown and explained. When the user needs to insert a child in the system, there is a form block ("New Child") to fill in. This block is illustrated in Figure 5 and it is composed by five fields: child's identification code, name, gender, and age. The last form to answer is whether the child is typically developing or not.

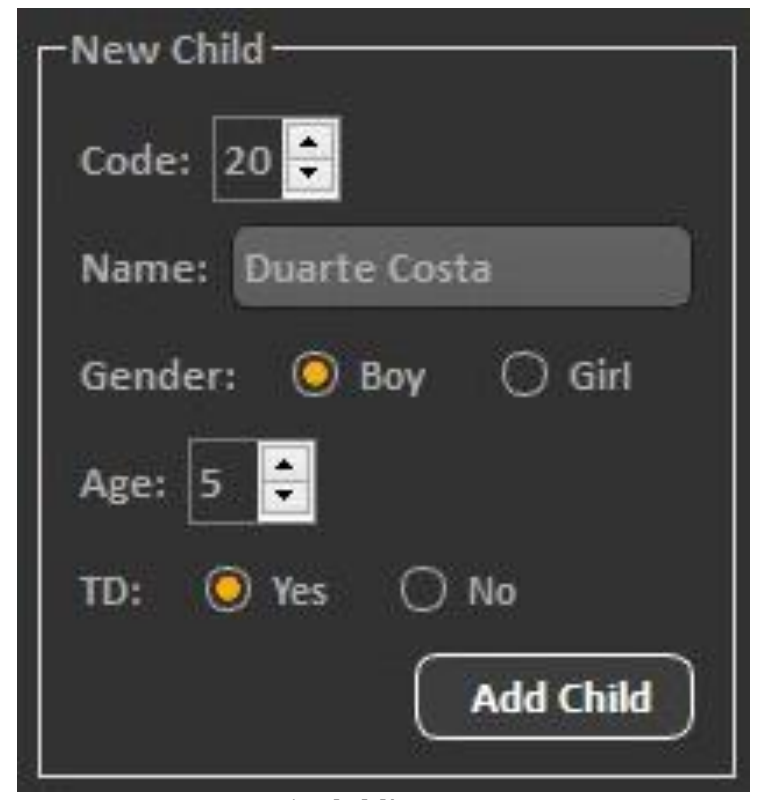

Figure 5: Child's registration.

After filling in all fields in the child's profile, it is necessary to press "Add Child" to add him/her in the system. Then, a new entry appears in the table in

Figure 6, which represents all the children in the database. The table presents, for each of all the registered children, the information profile and the number of sessions that he/she completed. Whenever a child is selected from the table, his/her profile is displayed as presented in Figure 7.

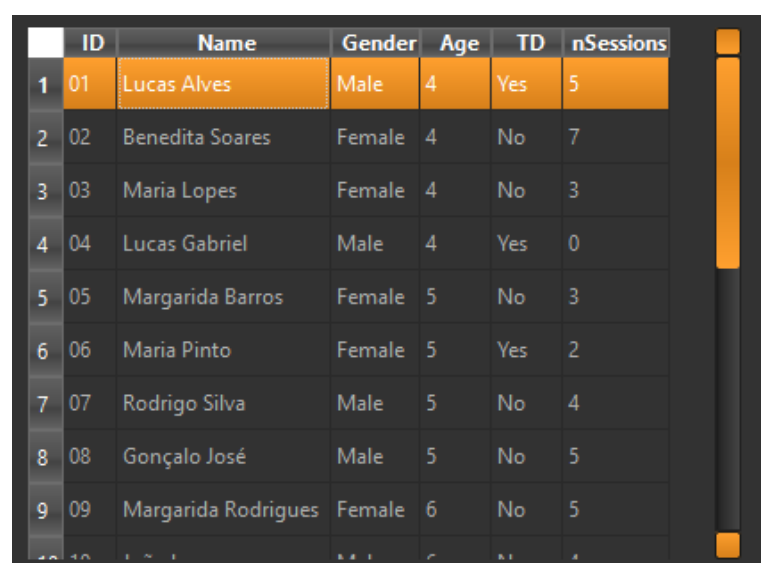

Figure 6: Child's registered and some information.

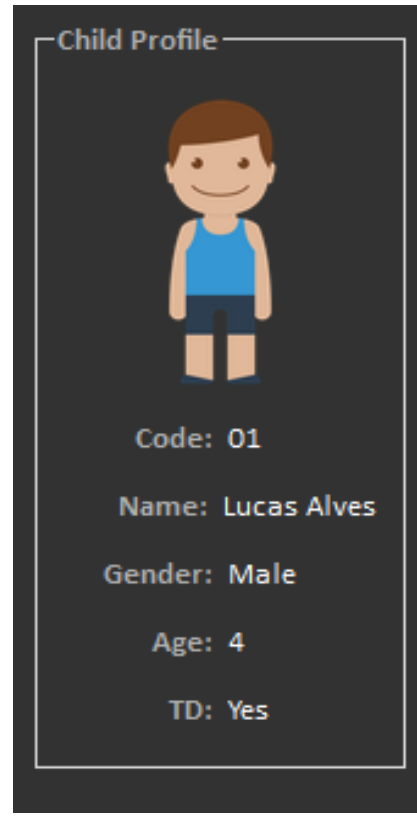

Figure 7: Child profile.

After selecting a child, the user can see his/her performance in the activities played. It is necessary to select which activity performance the user desires to see, as the child's performance is organized by activity. In Figure 8 it is presented the activity chosen, the first level of geometric figures $(F$ stands for geometric Figures and $C$ for Colours).

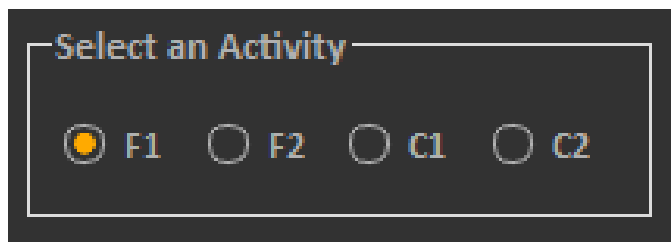

Figure 8: Selector of the activity performance ( $F$ stands for geometric Figures and C for Colours).

During the activity, all the answers are saved and when it stops, the information is compiled and organized. Besides that, the child's performance is calculated and then saved in the activity.

Figure 9 represents the child's performance in the geometric figures activity. This child performed twice the activity, and in each participation he/she correctly matched all the geometric figures, which is why all the bars have a $100 \%$ rate of success. Each colour of the graph bar corresponds to a geometric figure.

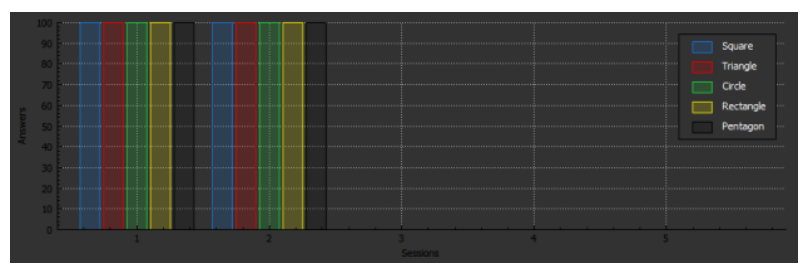

Figure 9: Child's performance - graph bars. 


\section{- Sessions}

The kindergarten's educators were asked to choose five children, those who had more difficulties of concentration and none or minor communication disorders, from each age group, i.e. five children with three-year-old, five with four-year-old and five with five-year-old. Each child performed both levels for both activities, and it was then given the opportunity to repeat one of the activities. Generally, in this case, the child chose to repeat the second level activity. The sessions were held in two days in a school room, where there were present some of the authors, to handle the developed system, the child and the kindergarten's educator.

When all the children suggested by the kindergarten's educators fulfilled the activities, the other children asked if they could perform an activity, because they would like to meet ZECA. Then, the children were organized into groups of five elements each and performed one of the second level activities. Thus, to each child it was assigned a game piece, from one of the activities. When a piece was requested by the robot, the child with this piece should place the piece on the game board.

\section{Preliminary Results}

The system was first tested in laboratorial environment to detect possible malfunctions. Light and time slots adjustments for recognizing the figure/colour were performed.

In order to detect system constraints in a school environment and to evaluate if the proposed system is a suitable tool to be used in kindergartens to promote academic and/or social skills, field tests were conducted with 15 children from 3 to 5 years old. Two children of this sample group presented social and concentration impairments. It is worth mentioning that the diagnosis of impairments in these young children is performed later where symptoms are undoubtedly detected. At this age, children are only signalized as a possible candidate to have a particular diagnosis. That is the reason to have only two children in the sample group signalized.

The main objective of the tests was to evaluate the robustness of the system as well its adequacy to be used in school environments. The analysis of the child's improvement in the learning process was not part of this study.

It was possible to infer the sensibility of the system regarding light intensity in the room as well as the necessity to design robust pieces (paperboard is not robust enough to be manipulated by children).

Figure 10 shows a scene of one of the performed tests. The child sat in front of the robot and the game table (with the geometric figures and colours) was placed between them.
The contact with the children was very positive, they were concentrated in the tasks and committed to carry out the proposed activities.

The most successful activities were the 2nd level of difficulty in each activity. The children asked to repeat these activities. Some of them even wanted to spend the day with ZECA.

Initially, the study was to be conducted with only 15 children (five from each group). But due to the impact caused by ZECA robot, all the children asked to have the opportunity to carry out the activities. They were then divided into several groups and each group performed an activity with ZECA. All children participated in the activity with great enthusiasm.

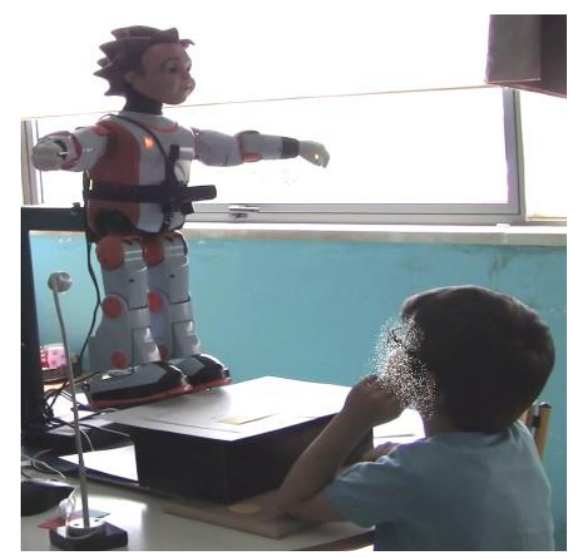

Figure 10: Test scenario.

\section{Final Comments}

Part of a proper care about those with special needs involves the development of new technologies and devices aiming at improving their quality of life. Academic research on this subject should be followed by the industrial development of commercial products and governmental institutions may play an important role by establishing conditions ensuring that the results are made available to those who need them.

This paper presented an interactive environment, still at the early stages of research level, developed in order to promote academic and social skills, and aimed at helping children with ASD. It uses ZECA, a humanoid robot Zeno $R-50$, acting as the promoter of the interaction with children, by teaching colours and geometric figures in the context of two educational game scenarios: recognition of five geometric shapes (square, triangle, rectangle, circle, and pentagon) and recognition of five colours (red, blue, green, black, and yellow). The robot establishes a dialogue with the child, congratulating the child when he/she hits the correct geometric figure or colour and/or transmitting positive reinforcement if the child fails.

The system includes a camera, a computer, and the robot. A communication protocol between the robot and the computer was developed. 
Image processing algorithms were implemented in order to detect the geometric figures and colours. The interface LCFZ (Learn Colours and Figures with ZECA) was created. LCFZ allows the main user to register the participant, to select the activity and also to obtain a record of all the information obtained during the accomplishment of the activity (as for example, the answers given by the participants as well as to obtain a graphic record of the performance in each activity).

The laboratorial environment allowed to detect the malfunctions and improve the system in terms of light and programming slot times adjustments.

The tests conducted in a kindergarten with 15 children from 3 to 5 years old allowed to ascertain the adequacy of the proposed tool to promote academic and/or social-

The children reacted very positively to this interactive environment, participated actively in the activities, even those who had difficulties in concentration.

The results obtained allow to conclude that the proposed system is able to interact with young children in a comfortable and natural way, giving a strong indication on the use of this particular system in the context of promoting academic and social skills. The next step in the research is to test the system with children with ASD.

\section{Acknowledgements}

The authors are grateful to the teachers and students of the kindergarten of Gualtar, Braga and their parents for their participation in the project. The authors also would like to express their acknowledgments to COMPETE: POCI-01-0145FEDER-007043 and FCT - Fundação para a Ciência e Tecnologia within the Project Scope: UID/CEC/00319/2013.

\section{References}

[1] A. Tapus, S. Member, and B. Scassellati, "The Grand Challenges in Socially Assistive Robotics," IEEE Robot. Autom. Mag., vol. 14, pp. 1-7, 2007.

[2] S. Fridenson-hayo et al., "Basic and Complex Emotion Recognition in Children with Autism: Cross Cultural Findings," Mol. Autism, pp. 1-11, 2016.

[3] S. Baron-Cohen and S. Wheelwright, "The empathy quotient: An investigation of adults with asperger syndrome or high functioning autism, and normal sex differences," J. Autism Dev. Disord., vol. 34, no. 2, pp. 163-175, 2004.
[4] F. Happé, J. Briskman, U. Frith, F. Happé, and U. Frith, "Exploring the cognitive phenotype of autism: weak 'central coherence' in parents and siblings of children with autism: II. Real-life skills and preferences.," J. Child Psychol. Psychiatry., vol. 42, no. 3, pp. 309-16, 2001.

[5] S. Costa, "Affective Robotics for Socio-Emotional Skills Development in Children with Autism Spectrum Disorders," University of Minho, 2014.

[6] E. S. Kim et al., "Social robots as embedded reinforcers of social behavior in children with autism," J. Autism Dev. Disord., vol. 43, no. 5, pp. 1038-1049, 2013.

[7] F. Michaud et al., "Autonomous spherical mobile robot for child-development studies," IEEE Trans. Syst. Man, Cybern. - Part A Syst. Humans, vol. 35, no. 4, pp. 471-480, 2005.

[8] D. J. Ricks and M. B. Colton, "Trends and considerations in robot-assisted autism therapy," Proc. - IEEE Int. Conf. Robot. Autom., pp. 43544359, 2010.

[9] S. Costa, H. Lehmann, B. Robins, K. Dautenhahn, and F. Soares, "Where is your nose?": developing body awareness skills among children with autism using a humanoid robot," ACHI 2013, Sixth Int. Conf. Adv. Comput. Interact., no. c, pp. 117-122, 2013.

[10] SofBank Robotics, "Discover Nao, the little humanoid robot from SoftBank Robotics | SoftBank Robotics." [Online]. Available: https://www.ald.softbankrobotics.com/en/coolrobots/nao. [Accessed: 01-Jun-2017].

[11] A. Griffiths, "The robot teacher connecting with autistic children - Telegraph," The Telegraph, 2014. [Online]. Available: http://www.telegraph.co.uk/technology/news/1 0632937/The-robot-teacher-connecting-withautistic-children.html. [Accessed: 17-Jun-2016].

[12] D. Mazzei et al., "Development and evaluation of a social robot platform for therapy in autism.," Conf. Proc. Annu. Int. Conf. IEEE Eng. Med. Biol. Soc. IEEE Eng. Med. Biol. Soc. Annu. Conf., vol. 2011, pp. 4515-8, 2011.

[13] P. Ekman et al., "Universals and cultural differences in the judgments of facial expressions of emotion.," Journal of Personality and Social Psychology, vol. 53, no. 4. American Psychological Association, US, pp. 712-717, 1987.

[14] K. Dautenhahn, "Design issues on interactive environments for children with autism.," 3Th Int. Conf. Disabil. , Virtual Real. Assoc. Technol., pp. 153-162, 2000.

[15] B. Scassellati, "How social robots will help us to diagnose, treat, and understand autism," Robot. Res., pp. 552-563, 2007.

[16] "Robokind | Advanced Social Robots," 2015. [Online]. Available: http://www.robokindrobots.com/. [Accessed: 01-May-2016]. 